\title{
THE EFFECTS OF CARBON DIOXIDE INHALATION UPON THE CEREBRAL BLOOD FLOW AND CEREBRAL OXYGEN CONSUMPTION IN VASCULAR DISEASE ${ }^{1}$
}

\author{
By PAUL NOVACK,? HENRY A. SHENKIN, LEONARD BORTIN, BERNARD \\ GOLUBOFF, AND ALVIN M. SOFFE WITH THE TECHNICAL ASSISTANCE OF \\ MrS. PETER BATSON AND MISS DORIS GOLDEN
}

\author{
(From the Departments of Neurosurgery, Research, and Medicine of the Albert Einstein Medical \\ Center, Southern Division, Phila., Penna.)
}

(Submitted for publication February 3, 1953; accepted April 17, 1953)

\begin{abstract}
Alterations of the cerebral circulation were for a long time presumed to reflect principally changes in the systemic circulation. Recently, a high degree of control of the cerebral blood flow has been demonstrated to be the result of intrinsic mechanisms. An important means of such intrinsic control results from the effects of changes in arterial partial pressure of carbon dioxide upon the cerebral vessels. An increased arterial partial pressure of carbon dioxide, such as is produced by the inhalation of 5 per cent carbon dioxide, results in a marked and selective cerebral vasodilatation in normal young individuals with a consequent increase in the cerebral blood flow (1). It appeared that the effect of the inhalation of carbon dioxide might provide a convenient tool for the study of the degree of vascular tone in cerebrovascular disease states. The present investigation was initiated with the purpose of using this method to gain insight into the relative contributions of vascular spasm and arteriosclerosis towards the production of an increased cerebrovascular resistance.
\end{abstract}

\section{METHODS}

The cerebral blood flow (CBF) was measured by the nitrous oxide technique (2). All patients were in the postabsorptive state and the recumbent position. Following the completion of a control flow, an interval of 15 minutes was allowed to elapse. The patient was then primed for five minutes with a gas mixture of the following composition: 5 per cent $\mathrm{CO}_{2}, 21$ per cent $\mathrm{O}_{2}, 74$ per cent $\mathrm{N}_{2}$. The mask was then rapidly switched to allow the patient to inhale a similar gas mixture, containing 15 per cent $\mathrm{N}_{2} \mathrm{O}$ and 59 per cent $\mathrm{N}_{2}$ in addition to 5 per cent $\mathrm{CO}_{2}$ and 21 per cent $\mathrm{O}_{2}$. In this manner the experimental flow was determined over a ten minute period.

\footnotetext{
1 Supported by a grant from the National Institutes of Health.

2 National Heart Institute Postdoctorate Research Fellow, 1952-1953.
}

The jugular venous pressure (JVP) was measured with a spinal fluid manometer using the level at which the vessel was entered as the " $O$ " reference point. In the supine position, this is approximately at the level of the right atrium. The mean arterial blood pressure (MABP) was measured in a femoral artery using a damped mercury manometer. The oxygen and carbon dioxide contents of the blood samples were determined by the method of van Slyke and Neill (3). Blood $\mathrm{pH}$ was determined anaerobically at room temperature with a glass electrode and corrected to body temperature using the formula of Rosenthal (4). The $\mathrm{pCO}_{2}$ was determined from standard nomograms (3).

\section{MATERIAL}

The subjects of this study have been divided into four groups according to criteria previously described $(5): 1$ ) Young normotensive individuals without evidence of arteriosclerosis with a mean age of $30 ; 2$ ) elderly, arteriosclerotic but normotensive individuals with a mean age of 64 ; 3) hypertensive individuals without evidence of arteriosclerosis with a mean age of 51 ; and, 4) hypertensive and arteriosclerotic individuals whose mean age was 55. Six of the latter group had histories of cerebrovascular accidents or mental deterioration. The presence of arteriosclerosis was diagnosed on the basis of historical or electrocardiographic evidence of coronary artery disease, vascular calcifications on X-ray, peripheral vascular ischemia, retinal changes, or a previous cerebrovascular accident. The control data for these and other groups have been presented and discussed in detail in another communication (5). The present report is concerned only with the responses to $\mathrm{CO}_{2}$ inhalation.

\section{RESULTS}

Table IA presents the cerebral circulatory data for Group 1. A 40 per cent increase in the CBF from the control value of $53 \mathrm{cc}$. per $100 \mathrm{gm}$. per min. to $74 \mathrm{cc}$. per $100 \mathrm{gm}$. per min. is noted. This increased blood flow is in part attributable to the 4 per cent increase in MABP but is mainly a reflection of the 33 per cent drop in CVR. The increase in JVP noted is a reflection of the increased 
TABLE IA

Effects of 5 per cent $\mathrm{CO}_{2}$ inhalation on cerebral circulatory functions of young, normotensive, non-arteriosclerotic individuals*

\begin{tabular}{|c|c|c|c|c|c|c|c|c|c|c|c|c|}
\hline \multirow{2}{*}{ Subject } & \multicolumn{2}{|c|}{ 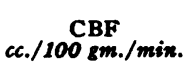 } & \multicolumn{2}{|c|}{$\begin{array}{c}\text { CVR } \\
\operatorname{mm} . \mathrm{Hg}_{\mathrm{g}} / \mathrm{cc} . / \\
100 \mathrm{gm} . / \mathrm{min} .\end{array}$} & \multicolumn{2}{|c|}{$\begin{array}{l}\mathrm{CMRO}_{2} \\
\text { cc./100 gm./min. }\end{array}$} & \multicolumn{2}{|c|}{$\underset{m m . ~}{\text { MABP }}$} & \multicolumn{2}{|c|}{$\underset{m m . ~}{J V P}$} & \multicolumn{2}{|c|}{ R. $\mathbf{Q}$. } \\
\hline & I & II & I & II & I & II & I & II & I & II & I & II \\
\hline $\begin{array}{l}\text { G. C. } \\
\text { S. W. } \\
\text { F. B. } \\
\text { S. G. } \\
\text { M. H. } \\
\text { D. M. } \\
\text { S. R. } \\
\text { A. H. } \\
\text { S. K. } \\
\text { E. G. } \\
\text { S. D. } \\
\text { D. C. }\end{array}$ & $\begin{array}{l}47 \\
77 \\
47 \\
35 \\
22 \\
54 \\
38 \\
52 \\
74 \\
54 \\
78 \\
57\end{array}$ & $\begin{array}{r}54 \\
89 \\
64 \\
48 \\
81 \\
78 \\
80 \\
64 \\
77 \\
61 \\
102 \\
92\end{array}$ & $\begin{array}{l}1.8 \\
1.0 \\
1.5 \\
2.1 \\
3.9 \\
1.3 \\
2.5 \\
2.0 \\
1.1 \\
1.7 \\
1.1 \\
1.3\end{array}$ & $\begin{array}{l}1.7 \\
0.9 \\
1.2 \\
1.6 \\
1.0 \\
0.9 \\
1.3 \\
1.7 \\
1.0 \\
1.4 \\
0.9 \\
0.9\end{array}$ & $\begin{array}{l}2.8 \\
4.2 \\
3.4 \\
2.5 \\
1.6 \\
2.9 \\
2.0 \\
4.8 \\
4.9 \\
2.7 \\
5.1 \\
3.8\end{array}$ & $\begin{array}{l}2.3 \\
2.3 \\
2.9 \\
2.5 \\
3.5 \\
3.6 \\
2.7 \\
3.8 \\
4.4 \\
2.1 \\
3.3 \\
3.8\end{array}$ & $\begin{array}{r}99 \\
84 \\
79 \\
81 \\
91 \\
79 \\
100 \\
111 \\
95 \\
100 \\
91 \\
83\end{array}$ & $\begin{array}{r}105 \\
90 \\
85 \\
85 \\
92 \\
82 \\
105 \\
111 \\
97 \\
94 \\
104 \\
90\end{array}$ & $\begin{array}{r}186 \\
90 \\
101 \\
98 \\
84 \\
98 \\
73 \\
61 \\
152 \\
108 \\
101 \\
80\end{array}$ & $\begin{array}{r}195 \\
88 \\
100 \\
126 \\
166 \\
130 \\
85 \\
67 \\
220 \\
112 \\
128 \\
100\end{array}$ & $\begin{array}{l}1.00 \\
0.93 \\
0.88 \\
0.97 \\
0.99 \\
1.29 \\
0.78 \\
1.06 \\
1.01 \\
0.92 \\
0.92 \\
0.98\end{array}$ & $\begin{array}{l}0.92 \\
0.78 \\
1.09 \\
0.96 \\
0.94 \\
1.16 \\
1.10 \\
0.95 \\
0.93 \\
1.04 \\
0.92 \\
1.00\end{array}$ \\
\hline Mean & 53 & $74 \dagger$ & 1.8 & $1.2 \dagger$ & 3.4 & 3.4 & 91 & $95 \dagger$ & 103 & $126 t$ & 0.98 & 0.98 \\
\hline
\end{tabular}

* CBF : Cerebral blood flow.

CVR: Cerebrovascular resistance $=\frac{\text { MABP }- \text { JVP }}{\text { CBF }}$.

† Significant change, $\mathrm{p}<.05$.

$\mathrm{CMRO}_{2}$ : Cerebral oxygen consumption.

MABP: Mean arterial blood pressure.

JVP: Jugular venous pressure.

R.Q.: Cerebral respiratory quotient.

I: Control value.

II : Value after inhalation of $5 \% \mathrm{CO}_{2}$.

CBF. The other cerebral circulatory functions are not significantly changed.

Table IB presents in detail the effects of the inhalation of 5 per cent $\mathrm{CO}_{2}$ upon the various blood constituents.
Table IIA presents the data concerned with the effects of 5 per cent $\mathrm{CO}_{2}$ inhalation upon the cerebral circulatory functions of the aged, arteriosclerotic, but normotensive group. The increase in $\mathrm{CBF}$ from $47 \mathrm{cc}$. per $100 \mathrm{gm}$. per min. to $55 \mathrm{cc}$.

TABLE IB

Effects of inhalation of 5 per cent $\mathrm{CO}_{2}$ on blood constituents of young, normotensive, non-arteriosclerotic individuals*

\begin{tabular}{|c|c|c|c|c|c|c|c|c|c|c|c|c|c|c|c|c|c|c|}
\hline \multirow{2}{*}{ Subject } & \multicolumn{2}{|c|}{$\begin{array}{c}\mathrm{AO}_{2} \\
\text { Vol. \% }\end{array}$} & \multicolumn{2}{|c|}{$\underset{\text { Vol. } \%}{\mathrm{ACO}_{2}}$} & \multicolumn{2}{|c|}{$\mathrm{ApH}$} & \multicolumn{2}{|c|}{$\underset{m m . \mathrm{Hg}_{\mathbf{g}}}{\mathrm{ApCO}_{2}}$} & \multicolumn{2}{|c|}{$\begin{array}{c}\mathrm{VO}_{2} \\
\text { Vol. \% }\end{array}$} & \multicolumn{2}{|c|}{$\begin{array}{l}\mathrm{VCO}_{2} \\
\text { Vol. } \%\end{array}$} & \multicolumn{2}{|c|}{$\mathrm{VpH}$} & \multicolumn{2}{|c|}{$\underset{m m . H_{g}}{\mathrm{VpCO}_{2}}$} & \multicolumn{2}{|c|}{$\underset{V o l . \%}{\left(\mathrm{~A}_{\%}\right.}$} \\
\hline & I & II & I & II & I & II & I & II & I & II & I & II & I & II & I & II & I & II \\
\hline $\begin{array}{l}\text { G. C. } \\
\text { S. W. } \\
\text { F. B. } \\
\text { S. G. } \\
\text { M. H. } \\
\text { D. M. } \\
\text { S. R. } \\
\text { A. H. } \\
\text { S. K. } \\
\text { E. G. } \\
\text { S. P. } \\
\text { D. O. }\end{array}$ & $\begin{array}{l}16.8 \\
14.9 \\
14.6 \\
19.4 \\
17.9 \\
18.8 \\
16.5 \\
15.4 \\
20.2 \\
15.0 \\
11.9 \\
12.1\end{array}$ & \begin{tabular}{|l|}
18.4 \\
15.3 \\
14.8 \\
19.9 \\
17.9 \\
19.3 \\
17.6 \\
15.1 \\
20.2 \\
14.9 \\
11.0 \\
12.1
\end{tabular} & $\begin{array}{l}47.3 \\
54.4 \\
40.1 \\
40.8 \\
39.6 \\
46.8 \\
51.4 \\
46.6 \\
50.9 \\
50.9 \\
48.5 \\
18\end{array}$ & $\begin{array}{l}48.9 \\
55.3 \\
44.7 \\
43.6 \\
46.8 \\
49.4 \\
52.5 \\
51.0 \\
51.8 \\
52.2 \\
51.6 \\
51.0\end{array}$ & $\begin{array}{l}7.33 \\
7.33 \\
7.31 \\
7.36 \\
7.42 \\
7.29 \\
7.34 \\
7.39 \\
7.39 \\
7.33 \\
7.38 \\
7.41\end{array}$ & $\begin{array}{l}7.25 \\
7.29 \\
7.25 \\
7.33 \\
7.33 \\
7.29 \\
7.31 \\
7.34 \\
7.33 \\
7.30 \\
7.28 \\
7.35\end{array}$ & $\begin{array}{l}46 \\
51 \\
39 \\
38 \\
32 \\
47 \\
48 \\
40 \\
45 \\
48 \\
42 \\
37\end{array}$ & $\begin{array}{l}56 \\
56 \\
49 \\
43 \\
45 \\
52 \\
53 \\
48 \\
51 \\
52 \\
53 \\
45\end{array}$ & $\begin{array}{r}10.8 \\
9.4 \\
7.3 \\
12.4 \\
10.6 \\
12.3 \\
11.3 \\
6.3 \\
13.5 \\
10.0 \\
5.3 \\
5.4\end{array}$ & $\begin{array}{r}12.2 \\
9.4 \\
4.5 \\
14.7 \\
13.5 \\
14.7 \\
14.2 \\
9.2 \\
14.4 \\
11.3 \\
8.8\end{array}$ & $\begin{array}{r}53.3 \\
9.4 \\
46.6 \\
47.7 \\
46.9 \\
55.3 \\
55.4 \\
56.3 \\
57.6 \\
55.5 \\
54.5 \\
54.7\end{array}$ & $\begin{array}{l}\mathbf{5 4 . 5} \\
59.9 \\
49.6 \\
48.6 \\
50.9 \\
54.7 \\
56.3 \\
56.6 \\
\mathbf{5 7 . 1} \\
\mathbf{5 5 . 8} \\
\mathbf{5 4 . 6}\end{array}$ & $\begin{array}{l}7.30 \\
7.30 \\
7.28 \\
7.33 \\
7.39 \\
7.28 \\
7.29 \\
7.39 \\
7.33 \\
7.29 \\
7.32 \\
7.35\end{array}$ & $\begin{array}{l}7.23 \\
7.28 \\
7.22 \\
7.30 \\
7.29 \\
7.28 \\
7.27 \\
7.33 \\
7.29 \\
7.27 \\
7.26 \\
7.32\end{array}$ & $\begin{array}{l}51 \\
55 \\
47 \\
40 \\
36 \\
57 \\
57 \\
48 \\
51 \\
54 \\
49 \\
46\end{array}$ & $\begin{array}{l}60 \\
60 \\
58 \\
47 \\
51 \\
59 \\
62 \\
53 \\
57 \\
60 \\
56 \\
50\end{array}$ & $\begin{array}{l}6.0 \\
5.4 \\
7.3 \\
7.1 \\
7.3 \\
6.6 \\
5.2 \\
9.1 \\
6.6 \\
5.0 \\
6.6 \\
6.7\end{array}$ & $\begin{array}{l}6.1 \\
5.9 \\
4.5 \\
5.3 \\
4.3 \\
4.6 \\
3.4 \\
6.0 \\
5.7 \\
3.5 \\
3.2 \\
4.1\end{array}$ \\
\hline Mean & 16.1 & 16.4 & 47.1 & $49.9+$ & 7.36 & $7.30+$ & 43 & $50+$ & 9.6 & $11.2 \dagger$ & 53.6 & $54.5 \dagger$ & 7.32 & $7.28 \dagger$ & 49 & $56+$ & 6.6 & $4.7 \dagger$ \\
\hline
\end{tabular}

* A: Arterial.

† Significant change, $\mathrm{p}<.05$.

V: Venous.

$\mathrm{O}_{2}$ : Oxygen content.

$\mathrm{CO}_{2}$ : Carbon dioxide content.

$\mathrm{pCO}_{2}$ : Partial pressure of carbon dioxide. 
TABLE IIA

Effects of inhalation of 5 per cent $\mathrm{CO}_{2}$ on cerebral circulatory functions of aged, normotensive, arteriosclerotic individuals*

\begin{tabular}{|c|c|c|c|c|c|c|c|c|c|c|c|c|}
\hline \multirow{2}{*}{ Subject } & \multicolumn{2}{|c|}{ cc. $/ 100 \mathrm{gm} . / \mathrm{min}}$. & \multicolumn{2}{|c|}{ 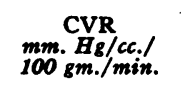 } & \multicolumn{2}{|c|}{$\begin{array}{l}\mathrm{CMRO}_{2} \\
\text { cc./100 gm./min. }\end{array}$} & \multicolumn{2}{|c|}{$\begin{array}{l}\text { MABP } \\
m m . H_{g}\end{array}$} & \multicolumn{2}{|c|}{$\underset{m m . H}{\mathrm{JVP}}$} & \multicolumn{2}{|c|}{ R. $Q$. } \\
\hline & I & II & I & II & I & II & I & II & I & II & I & II \\
\hline $\begin{array}{l}\text { M. D. } \\
\text { S. A. } \\
\text { H.S. } \\
\text { J. P. } \\
\text { E. G. } \\
\text { B. D. } \\
\text { E. G. } \\
\text { M. S. } \\
\text { S. K. } \\
\text { J. L. }\end{array}$ & $\begin{array}{l}48 \\
29 \\
38 \\
98 \\
54 \\
45 \\
33 \\
36 \\
51 \\
42\end{array}$ & $\begin{array}{l}46 \\
39 \\
39 \\
99 \\
63 \\
50 \\
42 \\
33 \\
68 \\
66\end{array}$ & $\begin{array}{l}2.0 \\
3.2 \\
2.6 \\
0.8 \\
1.7 \\
1.8 \\
2.4 \\
2.6 \\
1.3 \\
2.7\end{array}$ & $\begin{array}{l}2.3 \\
2.4 \\
2.8 \\
0.8 \\
1.6 \\
1.6 \\
1.8 \\
3.0 \\
1.1 \\
1.7\end{array}$ & $\begin{array}{l}3.3 \\
1.5 \\
3.0 \\
6.4 \\
3.9 \\
2.8 \\
1.0 \\
2.2 \\
3.5 \\
3.3\end{array}$ & $\begin{array}{l}3.6 \\
1.7 \\
1.9 \\
3.9 \\
3.9 \\
2.5 \\
1.8 \\
1.5 \\
3.2 \\
3.6\end{array}$ & $\begin{array}{r}100 \\
99 \\
100 \\
77 \\
98 \\
95 \\
86 \\
100 \\
72 \\
115\end{array}$ & $\begin{array}{r}110 \\
100 \\
113 \\
79 \\
111 \\
92 \\
79 \\
108 \\
76 \\
115\end{array}$ & $\begin{array}{r}65 \\
75 \\
38 \\
59 \\
98 \\
156 \\
77 \\
108 \\
54 \\
60\end{array}$ & $\begin{array}{r}70 \\
64 \\
67 \\
67 \\
105 \\
180 \\
82 \\
115 \\
58 \\
60\end{array}$ & $\begin{array}{l}0.89 \\
1.12 \\
1.01 \\
0.51 \\
0.92 \\
0.81 \\
0.83 \\
0.97 \\
0.96 \\
0.99\end{array}$ & $\begin{array}{l}0.71 \\
0.78 \\
0.88 \\
0.81 \\
1.16 \\
0.96 \\
1.09 \\
0.84 \\
0.81 \\
0.98\end{array}$ \\
\hline Mean & 47 & $55 \dagger$ & 2.1 & 1.9 & 3.1 & 2.7 & 94 & 98 & 79 & 87 & 0.90 & 0.90 \\
\hline
\end{tabular}

* See Tables IA and IB for definitions of symbols.

$\dagger$ Significant change, $p<.05$.

TABLE IIB

Effects of inhalation of 5 per cent $\mathrm{CO}_{2}$ on blood constituents of aged, normotensive, arteriosclerotic individuals*

\begin{tabular}{|c|c|c|c|c|c|c|c|c|c|c|c|c|c|c|c|c|c|c|}
\hline \multirow{2}{*}{ Subject } & \multicolumn{2}{|c|}{$\begin{array}{c}\mathrm{AO}_{2} \\
\text { Vol. \% }\end{array}$} & \multicolumn{2}{|c|}{$\underset{\text { Vol. } \%}{\mathrm{ACO}_{2}}$} & \multicolumn{2}{|c|}{$\mathbf{A p H}$} & \multicolumn{2}{|c|}{$\underset{m m . H_{g}}{\mathrm{ApCO}_{2}}$} & \multicolumn{2}{|c|}{$\begin{array}{c}\mathrm{VO}_{2} \\
\text { Vol. \% }\end{array}$} & \multicolumn{2}{|c|}{$\begin{array}{l}\mathrm{VCO}_{2} \\
\text { Vol. \% }\end{array}$} & \multicolumn{2}{|c|}{$\mathrm{VpH}$} & \multicolumn{2}{|c|}{$\underset{m m . H_{g}}{\mathrm{VpCO}_{2}}$} & \multicolumn{2}{|c|}{$\begin{array}{c}(\mathrm{A}-\mathrm{V}) \mathrm{O}, \\
\text { Vol. \% }\end{array}$} \\
\hline & I & II & I & II & I & II & I & II & I & II & I & II & I & II & I & II & I & II \\
\hline $\begin{array}{l}\text { M. D. } \\
\text { S. A. } \\
\text { H. S. } \\
\text { J. P. } \\
\text { E. G. } \\
\text { B. D. } \\
\text { E. Gi. } \\
\text { M. S. } \\
\text { S. K. } \\
\text { J. L. }\end{array}$ & $\begin{array}{l}18.5 \\
18.4 \\
17.0 \\
15.1 \\
19.8 \\
18.1 \\
19.5 \\
19.2 \\
16.7 \\
16.5\end{array}$ & $\begin{array}{l}18.8 \\
18.8 \\
15.9 \\
15.1 \\
20.6 \\
19.5 \\
20.5 \\
20.0 \\
16.8 \\
16.8\end{array}$ & $\begin{array}{l}48.1 \\
44.8 \\
60.7 \\
50.0 \\
46.3 \\
43.6 \\
42.5 \\
48.6 \\
47.4 \\
44.5\end{array}$ & $\begin{array}{l}51.4 \\
47.5 \\
64.6 \\
51.8 \\
47.0 \\
46.1 \\
41.8 \\
52.0 \\
49.8 \\
47.5\end{array}$ & $\begin{array}{l}7.31 \\
7.34 \\
7.35 \\
\overline{7.36} \\
- \\
7.31 \\
7.33 \\
7.39\end{array}$ & $\begin{array}{l}7.26 \\
7.30 \\
7.30 \\
\overline{7.34} \\
= \\
\overline{7.29} \\
7.31 \\
7.32\end{array}$ & $\begin{array}{l}47 \\
43 \\
56 \\
43 \\
- \\
49 \\
45 \\
38\end{array}$ & $\begin{array}{l}54 \\
50 \\
66 \\
\frac{47}{-} \\
\overline{57} \\
49 \\
47\end{array}$ & $\begin{array}{r}11.6 \\
13.4 \\
9.1 \\
8.6 \\
12.6 \\
12.0 \\
16.6 \\
13.5 \\
9.9 \\
8.8\end{array}$ & $\begin{array}{l}13.2 \\
14.3 \\
11.1 \\
11.2 \\
14.4 \\
14.5 \\
16.2 \\
15.4 \\
12.1 \\
11.3\end{array}$ & $\begin{array}{l}54.3 \\
50.4 \\
68.6 \\
55.3 \\
53.0 \\
48.5 \\
44.8 \\
54.5 \\
53.9 \\
52.1\end{array}$ & $\begin{array}{l}\mathbf{5 5 . 4} \\
50.9 \\
68.8 \\
55.0 \\
54.3 \\
50.8 \\
46.4 \\
\mathbf{5 5 . 9} \\
\mathbf{5 5 . 5} \\
\mathbf{5 2 . 9}\end{array}$ & $\begin{array}{l}7.27 \\
7.30 \\
7.30 \\
\overline{7.30} \\
- \\
7.29 \\
7.31 \\
7.33\end{array}$ & $\begin{array}{l}7.24 \\
7.28 \\
7.26 \\
\overline{7.28} \\
- \\
\overline{7.25} \\
7.27 \\
7.29\end{array}$ & $\begin{array}{l}55 \\
53 \\
70 \\
47 \\
- \\
57 \\
53 \\
46\end{array}$ & $\begin{array}{l}65 \\
56 \\
75 \\
59 \\
-\overline{-} \\
55 \\
52\end{array}$ & $\begin{array}{l}6.9 \\
5.0 \\
7.8 \\
6.5 \\
7.2 \\
6.1 \\
2.9 \\
5.7 \\
6.8 \\
7.7\end{array}$ & $\begin{array}{l}5.6 \\
4.5 \\
4.8 \\
3.9 \\
6.2 \\
5.0 \\
4.3 \\
4.6 \\
4.7 \\
5.5\end{array}$ \\
\hline Mean & 17.9 & 18.3 & 47.7 & $50.0 \dagger$ & 7.34 & $7.30+$ & 46 & $53+$ & 11.6 & $13.4 \dagger$ & 54.3 & $55.4 \dagger$ & 7.30 & $7.27+$ & 54 & $62+$ & 6.3 & $4.9 \dagger$ \\
\hline
\end{tabular}

* See Tables IA and IB for definitions of symbols.

† Significant change, $p<.05$.

TABLE IIIA

Effects of inhalation of 5 per cent $\mathrm{CO}_{2}$ on cerebral circulatory functions of individuals with essential hypertension uncomplicated by arteriosclerosis*

\begin{tabular}{|c|c|c|c|c|c|c|c|c|c|c|c|c|}
\hline \multirow{2}{*}{ Subject } & \multicolumn{2}{|c|}{$c c . / 100_{\mathrm{gm} .}^{\mathrm{CBF}} / \mathrm{min}}$. & \multicolumn{2}{|c|}{$\underset{100}{\operatorname{CVR}} \underset{\mathrm{gm} . / \mathrm{min} .}{\mathrm{H} / \mathrm{cc} . /}$} & \multicolumn{2}{|c|}{$\begin{array}{c}\mathrm{CMRO}_{2} \\
\text { cc./100 } \mathrm{gm.} / \min .\end{array}$} & \multicolumn{2}{|c|}{$\underset{\mathbf{m m} . \mathrm{Hg}}{\operatorname{MABP}}$} & \multicolumn{2}{|c|}{$\underset{m m .}{\mathrm{JVP}} \mathrm{H}_{\mathbf{0}} \mathrm{O}$} & \multicolumn{2}{|c|}{ R. $\mathbf{Q}$. } \\
\hline & I & II & I & II & I & II & I & II & I & II & I & II \\
\hline $\begin{array}{l}\text { B. B. } \\
\text { G. B. } \\
\text { C. H. } \\
\text { S. P. } \\
\text { D. B. } \\
\text { R. S. }\end{array}$ & $\begin{array}{l}50 \\
60 \\
47 \\
55 \\
67 \\
31\end{array}$ & $\begin{array}{r}72 \\
76 \\
82 \\
103 \\
78 \\
102\end{array}$ & $\begin{array}{l}2.5 \\
2.4 \\
3.2 \\
1.9 \\
1.9 \\
3.5\end{array}$ & $\begin{array}{l}1.9 \\
2.0 \\
2.3 \\
1.0 \\
1.7 \\
1.2\end{array}$ & $\begin{array}{l}3.1 \\
3.7 \\
3.3 \\
2.8 \\
3.7 \\
1.9\end{array}$ & $\begin{array}{l}1.2 \\
3.5 \\
4.9 \\
4.5 \\
3.4 \\
2.0\end{array}$ & $\begin{array}{l}133 \\
154 \\
160 \\
115 \\
132 \\
121\end{array}$ & $\begin{array}{l}151 \\
160 \\
200 \\
110 \\
140 \\
137\end{array}$ & $\begin{array}{l}120 \\
137 \\
160 \\
120 \\
107 \\
165\end{array}$ & $\begin{array}{l}145 \\
160 \\
200 \\
125 \\
107 \\
260\end{array}$ & $\begin{array}{l}0.98 \\
1.09 \\
0.77 \\
0.79 \\
0.81 \\
1.15\end{array}$ & $\begin{array}{l}0.95 \\
0.92 \\
0.96 \\
0.83 \\
0.86 \\
2.06\end{array}$ \\
\hline Mean & 52 & $86 t$ & 2.6 & $1.7 \dagger$ & 3.1 & 3.6 & 136 & $150 \dagger$ & 135 & $166 \dagger$ & 0.93 & 1.10 \\
\hline
\end{tabular}

* See Tables IA and IB for definitions of symbols.

† Significant change, $p<.05$. 
TABLE IIIB

Effects of inhalation of 5 per cent $\mathrm{CO}_{2}$ on blood constituents of individuals with essential hypertension uncomplicated by arteriosclerosis*

\begin{tabular}{|c|c|c|c|c|c|c|c|c|c|c|c|c|c|c|c|c|c|c|}
\hline \multirow{2}{*}{ Subject } & \multicolumn{2}{|c|}{$\begin{array}{c}\mathrm{AO}_{\mathbf{2}} \\
\mathrm{Vol.} \%\end{array}$} & \multicolumn{2}{|c|}{$\begin{array}{c}\mathrm{ACO}_{2} \\
\text { Vol. \% }\end{array}$} & \multicolumn{2}{|c|}{$\mathrm{ApH}$} & \multicolumn{2}{|c|}{$\underset{m m . H_{g}}{\mathrm{ApCO}_{2}}$} & \multicolumn{2}{|c|}{$\begin{array}{c}\mathrm{VO}_{2} \\
\text { Vol. \% }\end{array}$} & \multicolumn{2}{|c|}{$\begin{array}{l}\mathrm{VCO}_{2} \\
\mathrm{Vol.} \%\end{array}$} & \multicolumn{2}{|c|}{$\mathrm{VpH}$} & \multicolumn{2}{|c|}{$\begin{array}{l}\mathrm{VpCO}_{2} \\
\mathbf{m m} . \mathrm{H}_{\mathrm{g}}\end{array}$} & \multicolumn{2}{|c|}{ 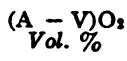 } \\
\hline & I & II & I & II & I & II & I & II & I & II & I & II & I & II & I & II & I & II \\
\hline $\begin{array}{l}\text { B. B. } \\
\text { G. B. } \\
\text { C. H. } \\
\text { S. P. } \\
\text { D. B. } \\
\text { R. S. }\end{array}$ & $\begin{array}{l}15.2 \\
16.6 \\
16.5 \\
15.7 \\
16.2 \\
16.0\end{array}$ & $\begin{array}{l}15.5 \\
17.6 \\
17.0 \\
14.1 \\
16.4 \\
15.0\end{array}$ & $\begin{array}{l}48.6 \\
45.7 \\
54.9 \\
48.5 \\
52.2\end{array}$ & $\begin{array}{l}50.6 \\
48.8 \\
56.4 \\
51.6 \\
53.1 \\
53.6\end{array}$ & $\begin{array}{l}7.44 \\
7.41 \\
7.38 \\
7.39 \\
7.41 \\
7.40\end{array}$ & $\begin{array}{l}7.43 \\
7.33 \\
7.33 \\
7.35 \\
7.34 \\
7.32\end{array}$ & $\begin{array}{l}37 \\
37 \\
47 \\
41 \\
42 \\
37\end{array}$ & $\begin{array}{l}40 \\
47 \\
54 \\
46 \\
49 \\
52\end{array}$ & $\begin{array}{r}9.1 \\
10.4 \\
9.5 \\
9.6 \\
10.7 \\
10.0\end{array}$ & $\begin{array}{r}11.1 \\
13.2 \\
11.0 \\
9.8 \\
12.0 \\
16.9\end{array}$ & $\begin{array}{l}54.6 \\
58.4 \\
60.3 \\
52.6\end{array}$ & $\begin{array}{l}54.9 \\
53.0 \\
62.1 \\
55.2 \\
56.9 \\
49.7\end{array}$ & $\begin{array}{l}7.35 \\
7.33 \\
7.35 \\
7.31 \\
7.33 \\
7.36\end{array}$ & $\begin{array}{l}7.34 \\
7.29 \\
7.32 \\
7.30 \\
7.20\end{array}$ & $\begin{array}{l}47 \\
49 \\
52 \\
51 \\
51 \\
43\end{array}$ & 59 & $\begin{array}{l}6.1 \\
6.2 \\
7.1 \\
5.1 \\
5.5 \\
6.0\end{array}$ & $\begin{array}{l}4.5 \\
4.4 \\
6.0 \\
4.3 \\
4.4 \\
1.9\end{array}$ \\
\hline Mean & 15.9 & 15.9 & 49.1 & $52.4 \dagger$ & 7.41 & $7.35 \dagger$ & 40 & $48+$ & 9.9 & $12.3 \dagger$ & 54.7 & $55.3 \dagger$ & 7.34 & $7.31 \dagger$ & 49 & 54 & 6.0 & 4.3 \\
\hline
\end{tabular}

* See Tables IA and IB for definitions of symbols.

† Significant change, $p<.05$.

TABLE IVA

Effects of inhalation of 5 per cent $\mathrm{CO}_{2}$ on cerebral circulatory functions of hypertensive arteriosclerotic individuals*

\begin{tabular}{|c|c|c|c|c|c|c|c|c|c|c|c|c|}
\hline \multirow{2}{*}{ Subject } & \multicolumn{2}{|c|}{$\underset{c c . / 100 \mathrm{gm} . / \mathrm{min} .}{\mathrm{CBF}}$} & \multicolumn{2}{|c|}{ 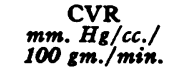 } & \multicolumn{2}{|c|}{$\underset{c c . / 100 \mathrm{gm} . / \min .}{\mathrm{CMRO}_{2}}$} & \multicolumn{2}{|c|}{$\underset{m m . H_{g}}{\text { MABP }}$} & \multicolumn{2}{|c|}{$\underset{m m . H_{2} O}{J V P}$} & \multicolumn{2}{|c|}{ R. $Q$. } \\
\hline & I & II & I & II & I & II & I & II & I & II & I & II \\
\hline $\begin{array}{l}\text { M. S. } \\
\text { S. F. } \\
\text { E. S. } \\
\text { S. L. } \\
\text { S. S. } \\
\text { C. K. } \\
\text { S. G. } \\
\text { D. K. } \\
\text { J. L. } \\
\text { M. W. }\end{array}$ & $\begin{array}{l}33 \\
33 \\
51 \\
41 \\
23 \\
29 \\
34 \\
33 \\
41 \\
44\end{array}$ & $\begin{array}{l}39 \\
46 \\
32 \\
94 \\
24 \\
48 \\
60 \\
46 \\
50 \\
54\end{array}$ & $\begin{array}{l}3.5 \\
3.9 \\
2.5 \\
3.0 \\
6.3 \\
4.9 \\
3.3 \\
3.4 \\
3.7 \\
2.6\end{array}$ & $\begin{array}{l}3.1 \\
2.6 \\
4.1 \\
1.4 \\
6.0 \\
3.6 \\
2.1 \\
2.0 \\
3.2 \\
2.1\end{array}$ & $\begin{array}{l}2.4 \\
2.6 \\
2.6 \\
2.8 \\
2.5 \\
2.6 \\
2.1 \\
2.6 \\
2.7 \\
3.7\end{array}$ & $\begin{array}{l}2.6 \\
1.6 \\
1.9 \\
5.2 \\
2.6 \\
2.1 \\
2.2 \\
2.6 \\
3.1\end{array}$ & $\begin{array}{l}120 \\
132 \\
141 \\
123 \\
140 \\
143 \\
117 \\
120 \\
156 \\
125\end{array}$ & $\begin{array}{l}125 \\
130 \\
145 \\
133 \\
150 \\
172 \\
135 \\
100 \\
169 \\
128\end{array}$ & $\begin{array}{r}60 \\
54 \\
210 \\
59 \\
65 \\
35 \\
55 \\
100 \\
78 \\
145\end{array}$ & $\begin{array}{r}70 \\
130 \\
198 \\
71 \\
80 \\
49 \\
110 \\
114 \\
90 \\
170\end{array}$ & $\begin{array}{l}0.98 \\
1.05 \\
1.27 \\
0.88 \\
0.86 \\
0.99 \\
1.11 \\
0.88 \\
0.77 \\
0.93\end{array}$ & $\begin{array}{l}0.95 \\
0.80 \\
0.72 \\
1.02 \\
-\overline{0.90} \\
1.11 \\
0.93 \\
1.30 \\
0.80\end{array}$ \\
\hline Mean & 36 & $49 \dagger$ & 3.7 & $3.0 \dagger$ & 2.6 & 2.7 & 132 & $139 \dagger$ & 86 & $108 t$ & 0.97 & 0.90 \\
\hline
\end{tabular}

* See Tables IA and IB for definitions of symbols.

$\uparrow$ Significant change, $p<.05$.

TABLE IVB

Effects of inhalation of 5 per cent $\mathrm{CO}_{2}$ on blood constituents of hypertensive arteriosclerotic individuals*

\begin{tabular}{|c|c|c|c|c|c|c|c|c|c|c|c|c|c|c|c|c|c|c|}
\hline \multirow{2}{*}{ Subject } & \multicolumn{2}{|c|}{$\begin{array}{c}\mathrm{AO}_{2} \\
\text { Vol. \% }\end{array}$} & \multicolumn{2}{|c|}{$\underset{\text { Vol. } \%}{\mathrm{ACO}_{2}}$} & \multicolumn{2}{|c|}{$\mathrm{ApH}$} & \multicolumn{2}{|c|}{$\underset{\mathrm{mm} . \mathrm{Hg}_{\mathrm{g}}}{\mathrm{ApCO}}$} & \multicolumn{2}{|c|}{$\begin{array}{c}\mathrm{VO}_{2} \\
\text { Vol. \% }\end{array}$} & \multicolumn{2}{|c|}{$\underset{\text { Vol. \% }}{\mathrm{VCO}_{2}}$} & \multicolumn{2}{|c|}{$\mathrm{VpH}$} & \multicolumn{2}{|c|}{$\underset{m m . \mathrm{Hg}_{\mathrm{g}}}{\mathrm{VpCO}_{\mathbf{p}}}$} & \multicolumn{2}{|c|}{$\underset{\text { Vol. \% }}{\left(\mathrm{A}-\mathrm{V}_{2}\right.}$} \\
\hline & I & II & I & II & I & II & I & II & I & II & I & II & I & II & I & II & I & II \\
\hline $\begin{array}{l}\text { M. S. } \\
\text { S. F. } \\
\text { E. S. } \\
\text { S. L. } \\
\text { S. S. } \\
\text { C. K. } \\
\text { S. G. } \\
\text { D. K. } \\
\text { J. L. } \\
\text { M. W. }\end{array}$ & \begin{tabular}{|l|}
21.2 \\
16.8 \\
19.2 \\
19.0 \\
22.5 \\
19.1 \\
10.8 \\
17.4 \\
19.6 \\
19.2 \\
\end{tabular} & \begin{tabular}{|l}
21.2 \\
14.8 \\
21.6 \\
19.1 \\
18.4 \\
10.9 \\
17.3 \\
19.6 \\
18.9
\end{tabular} & $\begin{array}{l}40.5 \\
45.6 \\
44.9 \\
48.1 \\
54.1 \\
37.8 \\
39.9 \\
43.8 \\
50.0 \\
49.1\end{array}$ & $\begin{array}{l}43.4 \\
53.8 \\
48.1 \\
49.1 \\
47.8 \\
46.3 \\
49.7 \\
48.9 \\
51.5\end{array}$ & $\begin{array}{l}7.37 \\
7.30 \\
7.38 \\
7.39 \\
7.51 \\
\overline{7.50} \\
7.43 \\
7.41\end{array}$ & $\begin{array}{l}7.30 \\
\overline{7.25} \\
7.36 \\
\overline{7.33} \\
\overline{7.36} \\
7.37 \\
7.37\end{array}$ & $\begin{array}{l}38 \\
47 \\
42 \\
47 \\
22 \\
27 \\
40 \\
41\end{array}$ & $\begin{array}{l}\frac{47}{57} \\
\frac{47}{46} \\
\frac{45}{45} \\
46\end{array}$ & $\begin{array}{r}13.9 \\
8.8 \\
14.1 \\
12.3 \\
10.1 \\
10.2 \\
4.7 \\
9.6 \\
13.0 \\
10.9\end{array}$ & \begin{tabular}{r|}
14.7 \\
11.4 \\
15.6 \\
13.5 \\
13.5 \\
7.3 \\
12.4 \\
14.4 \\
13.2
\end{tabular} & $\begin{array}{l}47.6 \\
53.8 \\
51.3 \\
54.1 \\
64.7 \\
46.6 \\
46.6 \\
50.7 \\
54.9 \\
56.9\end{array}$ & $\begin{array}{l}49.6 \\
56.5 \\
52.5 \\
54.8 \\
52.3 \\
50.3 \\
54.2 \\
55.8 \\
56.1\end{array}$ & $\begin{array}{l}7.32 \\
7.27 \\
7.33 \\
7.37 \\
7.41 \\
\overline{7.44} \\
7.39 \\
7.37\end{array}$ & $\begin{array}{l}7.27 \\
\overline{7.24} \\
7.33 \\
\overline{7.33} \\
\overline{7.32} \\
7.32 \\
7.32\end{array}$ & $\begin{array}{l}48 \\
55 \\
52 \\
59 \\
38 \\
39 \\
45 \\
47\end{array}$ & $\begin{array}{l}\frac{56}{63} \\
53 \\
\frac{54}{52} \\
54 \\
54\end{array}$ & \begin{tabular}{|r|}
7.3 \\
8.0 \\
5.0 \\
6.8 \\
12.3 \\
8.9 \\
6.0 \\
7.8 \\
6.7 \\
8.4
\end{tabular} & $\begin{array}{l}6.5 \\
3.4 \\
6.0 \\
5.6 \\
5.0 \\
3.6 \\
4.8 \\
5.2 \\
5.8\end{array}$ \\
\hline Mean & 18.5 & 18.0 & 45.4 & $48.7 \dagger$ & 7.41 & $7.33 t$ & 38 & $48 \dagger$ & 10.8 & $12.9 \dagger$ & 52.7 & $53.6 \dagger$ & 7.36 & $7.30 \dagger$ & 48 & $55 \dagger$ & 7.7 & $5.1 \dagger$ \\
\hline
\end{tabular}

* See Tables IA and IB for definitions of symbols.

† Significant change, $p<.05$. 
per $100 \mathrm{gm}$. per min. is significant $(\mathrm{p}<.05)$ but is less than for Group 1 ( 17 per cent vs. 40 per cent). The increased $\mathrm{CBF}$ apparently results from the combination of an increase in the MABP from $94 \mathrm{~mm}$. $\mathrm{Hg}$ to $98 \mathrm{~mm}$. $\mathrm{Hg}$ and a slight drop in the CVR, from $2.1 \mathrm{~mm}$. $\mathrm{Hg}$ per cc. per $100 \mathrm{gm}$. per min. to $1.9 \mathrm{~mm}$. $\mathrm{Hg}$ per cc. per $100 \mathrm{gm}$. per min., although neither of these changes is statistically significant in itself. Table IIB summarizes the data for the effects of 5 per cent $\mathrm{CO}_{2}$ inhalation upon the blood constituents of the individuals in this group. The changes noted with respect to blood $\mathrm{CO}_{2}$ contents, $\mathrm{pH}$, and $\mathrm{pCO}_{2}$ are of the same magnitude as in Group 1.

Table IIIA presents the data illustrating the effects of 5 per cent $\mathrm{CO}_{2}$ inhalation upon the cerebral circulation of hypertensive, non-arteriosclerotic individuals. The 65 per cent increase in CBF from $52 \mathrm{cc}$. per $100 \mathrm{gm}$. per min. to $86 \mathrm{cc}$. per $100 \mathrm{gm}$. per min. is greater, but not significantly so, than that noted for Group 1. This increased CBF is attributable to the combination of a 34 per cent drop in the CVR and a 10 per cent increase in the MABP. Table IIIB summarizes the blood constituent data for the group.

Table IVA presents cerebral circulatory data for the hypertensive, arteriosclerotic patients indicating the effects of 5 per cent $\mathrm{CO}_{2}$ inhalation. On a percentage basis, the 36 per cent increase in the CBF from the greatly reduced control value is comparable to that noted for Group 1 ( 40 per cent) and somewhat less than that noted for Group 3 (65 per cent). However, the final level obtained is far below that noted in the two aforementioned groups. The $0.7 \mathrm{~mm}$. $\mathrm{Hg}$ per cc. per $100 \mathrm{gm}$. per min. drop in CVR noted for this group is, in terms of absolute units, similar to the decreases in CVR described for Groups 1 and 3. This is, however, only a 19 per cent decrease from the control CVR, as compared to the 33 per cent and 34 per cent decreases in CVR for Groups 1 and 3 respectively. Table IVB summarizes the blood constituent data for Group 4. The increases in $\mathrm{pCO}_{2}$ obtained with 5 per cent $\mathrm{CO}_{2}$ inhalation are of the same order as noted for the previous groups.

\section{DISCUSSION}

Vascular resistance is principally a function of blood viscosity and the diameters of the blood channels, particularly the arteriolar tree. There is no reason to believe there were differences in blood viscosity among the various groups. Changes in vascular resistance caused by an increased arterial $\mathrm{pCO}_{2}$ largely reflect variations in the diameters of the vessels. A diminished arteriolar diameter, with its consequent increased vascular resistance, may in turn be the result of either organic occlusive changes or functional vasoconstriction.

In considering the arteriosclerotic but normotensive group of elderly individuals, the control values for the various cerebral circulatory functions were found to be within normal limits. It was only when these patients were subjected to an increased arterial $\mathrm{pCO}_{2}$ that significant $\mathrm{ab}$ normalities of their cerebral vasculatures were unmasked. Unlike the normal response, little decrease in CVR occurred with the elevation of the arterial $\mathrm{pCO}_{2}$. The failure to obtain cerebrovascular relaxation in the presence of arteriosclerosis may be explained by several possibilities: 1) The vessels may be so rigid that they can neither dilate nor constrict to any stimulus; 2) the vessels in the individuals of this group may be already maximally dilated as a compensatory response to the development of arteriosclerosis; 3 ) arteriosclerotic vessels may no longer respond to the specific dilating stimulus of an increased arterial $\mathrm{pCO}_{2}$. The finding that the CVR of patients having combined hypertension and arteriosclerosis (Group 4) may be lowered by $\mathrm{CO}_{2}$ inhalations demonstrates that the cerebral vessels of arteriosclerotic individuals are sensitive to an increased arterial $\mathrm{pCO}_{2}$ and that such vessels still have a residual elasticity. This favors the second of the above possibilities as an explanation for the failure of $\mathrm{CO}_{2}$ inhalations to lower the CVR in the normotensive, arteriosclerotic group.

The possibility that $\mathrm{CO}_{2}$ inhalation increases extracerebral contamination of internal jugular venous blood specimens has been considered as a factor which might influence the results. Study of the curves in the various groups failed to reveal evidence of such an occurrence in a significant degree. The ten minute equilibration period for nitrous oxide was found adequate in all groups both before and after $\mathrm{CO}_{2}$ inhalation.

Various measures, including the inhalation of carbon dioxide, have been recommended to stimulate the cerebral blood flow in patients who have suffered a cerebral thrombosis. When the throm- 
bosis is the result of arteriosclerotic narrowing in the absence of hypertension, it would seem from the results obtained in this study that such efforts would be doomed to failure. Furthermore, if such patients are in incipient or actual cardiac decompensation, inhalation of 5 per cent $\mathrm{CO}_{2}$ has been found to embarrass further the general circulation and in many instances to decrease the cerebral blood flow (6). However, no final conclusion can be reached relative to the efficacy of such measures without a direct investigation of the cerebral circulation during the acute phase of a cerebral thrombosis. At such times cerebral vasoconstriction mediated by the autonomic system could presumably occur even in the absence of hypertension.

In uncomplicated hypertension, the increased cerebrovascular resistance is generally considered to be primarily the result of increased vascular tone rather than of arteriosclerotic narrowing of the vessels. Various measures which reduce the increased cerebrovascular resistance have been studied. Sympathectomy has been found to result in an 18 per cent decrease ( 7$)$, high spinal anesthesia, in a 16 per cent decrease (8), and dihydroergocornine, in a 32 per cent decrease (9) in the cerebrovascular resistance in essential hypertension. In the present study, the inhalation of 5 per cent $\mathrm{CO}_{2}$ produced a mean decrease in the cerebrovascular resistance of 35 per cent, revealing $\mathrm{CO}_{2}$ to be as powerful a cerebral vasodilator in essential hypertension as any of the previously studied methods. In addition, it is to be noted that this reduction in CVR resulting from an increased arterial $\mathrm{PCO}_{2}$ is not associated with a fall in the head of pressure as are the other methods. Even after the reduction in cerebrovascular resistance produced by the inhalation of 5 per cent $\mathrm{CO}_{2}$, the cerebrovascular resistance of patients with essential hypertension was $1.7 \mathrm{~mm}$. $\mathrm{Hg}$ per cc. per 100 gm. per min. as compared to the value of $1.2 \mathrm{~mm}$. $\mathrm{Hg}$ per cc. per $100 \mathrm{gm}$. per min. for the "normal" group after $\mathrm{CO}_{2}$ inhalation. This residual increased vascular resistance in the hypertensive group may be attributed to either a failure of $\mathrm{CO}_{2}$ to produce maximal vasodilatation, or to the existence of clinically inapparent arteriosclerosis in this group, or, to a combination of the two. The failure of such measures as sympathectomy, high spinal anesthesia, or dihydroergocornine to produce a greater cerebrovascular relaxation than is obtained with $\mathrm{CO}_{2}$ suggests that near maximal vasodilatation is obtained with $\mathrm{CO}_{2}$ inhalation, and that the residual increased cerebrovascular resistance may be due to organic occlusive disease. The possibility of directly measuring the vascular resistance of an organ and roughly dissociating its components attributable to functional spasm and organic occlusion may offer a test for predicting the maximal efficacy of therapeutic measures designed to relieve increased vascular resistance.

The above concept may be applied to the interpretation of the data for the group of cases possessing hypertension and clinically apparent arteriosclerosis. Increase of the arterial $\mathrm{pCO}_{2}$ in this group resulted in the lowering of the cerebrovascular resistance from $3.7 \mathrm{~mm}$. $\mathrm{Hg}$ per cc. per $100 \mathrm{gm}$. per $\mathrm{min}$. to $3.0 \mathrm{~mm}$. $\mathrm{Hg}$ per cc. per 100 gm. per min. The high residual cerebrovascular resistance suggests that the arteriosclerosis present in this group is considerably greater than in any of the previously discussed groups. This is the same conclusion previously derived (5).

From these data there seems to emerge some clarification of the interrelated effects of hypertension and arteriosclerosis upon the cerebral hemodynamics. In hypertension uncomplicated by clinically evident arteriosclerosis, the cerebrovascular resistance is increased, mostly due to increased arteriolar tone, but in part probably due to arteriosclerosis. This increase in cerebrovascular resistance parallels the increase in arterial pressure and there is no reduction in the cerebral blood flow. In arteriosclerosis without hypertension, a normal cerebral blood flow and cerebrovascular resistance tend to be maintained, possibly by some relaxation of the normal vascular tone. Finally, where hypertension and arteriosclerosis coexist, the arteriosclerosis seems to be of a much greater severity and diffuseness than that occurring in the absence of hypertension, and an increased vascular tone persists. The net effect is a great increase in the cerebrovascular resistance and a marked reduction in the cerebral blood flow and oxygen consumption.

\section{CONCLUSIONS}

1. The effects of inhalation of 5 per cent $\mathrm{CO}_{2}$ upon the cerebral circulations of individuals with varying degrees of vascular disease have been studied.

2. The normal response to an elevation of arterial $\mathrm{pCO}_{2}$ is a slight increase in the mean ar- 
terial blood pressure and a drop in cerebrovascular resistance with a consequent increase in cerebral blood flow.

3. A group composed of normotensive arteriosclerotic individuals failed to show a significant decrease of the cerebrovascular resistance, although the cerebral blood flow increased slightly.

4. A small group of individuals with uncomplicated essential hypertension were found to respond normally to $\mathrm{CO}_{2}$ inhalation. The decrease in cerebrovascular resistance noted in this group was as great as had previously been produced with other methods.

5. A group of hypertensive arteriosclerotic patients also responded normally to $\mathrm{CO}_{2}$ inhalation. The residual cerebrovascular resistance after $\mathrm{CO}_{2}$ vasodilatation was still markedly elevated above normal.

6. It is suggested that the response to $\mathrm{CO}_{2}$ inhalation provides a convenient means for dissociating and estimating the components of an increased cerebrovascular resistance attributable to functional vasoconstriction and arteriosclerotic narrowing to the lumens of the vessels.

\section{REFERENCES}

1. Kety, S. S., and Schmidt, C. F., The effects of altered arterial tensions of carbon dioxide and oxygen on cerebral blood flow and cerebral oxygen consumption of normal young men. J. Clin. Invest., 1948, $27,484$.

2. Kety, S. S., and Schmidt, C. F., The nitrous oxide method for the quantitative determination of cerebral blood flow in man: Theory, procedure, and normal values. J. Clin. Invest., 1948, 27, 476.

3. Peters, J. P., and van Slyke, D. D., Quantitative Clinical Chemistry. Vol. II, Methods, Williams \& Wilkins, Balt., 1932.

4. Rosenthal, T. B., The effect of temperature on the $\mathrm{pH}$ of blood and plasma in vitro. J. Biol. Chem., 1948, $173,25$.

5. Shenkin, H. A., Novack, P., Goluboff, B., Soffe, A. M., and Bortin, $L$., The effects of aging, arteriosclerosis and hypertension upon the cerebral circulation. J. Clin. Invest., 1953, 32, 459.

6. Shenkin, H. A., Novack, P., and Bortin, L., Unpublished observations.

7. Shenkin, H. A., Hafkenschiel, J. H., and Kety, S. S., Effects of sympathectomy on the cerebral circulation of hypertensive patients. Arch. Surg., 1950, 61, 319.

8. Kety, S. S., King, B. D., Horvath, S. M., Jeffers, W. A., and Hafkenschiel, J. H., The effects of an acute reduction in blood pressure by means of differential spinal sympathetic block on the cerebral circulation of hypertensive patients. J. Clin. Invest., 1950, 29, 402.

9. Hafkenschiel, J. H., Crumpton, C. W., Moyer, J. H., and Jeffers, W. A., The effects of dihydroergocornine on the cerebral circulation of patients with essential hypertension. J. Clin. Invest., 1950, 29, 408. 